\title{
Application of Near-Infrared Spectroscopy to Quantitatively Determine Relative Content of Puccnia striiformis f. sp. tritici DNA in Wheat Leaves in Incubation Period
}

\author{
Yaqiong Zhao, ${ }^{1}$ Yilin Gu, ${ }^{1}$ Feng Qin, ${ }^{1}$ Xiaolong $\mathrm{Li},{ }^{1}$ Zhanhong Ma, ${ }^{1}$ Longlian Zhao, ${ }^{2}$ \\ Junhui Li, ${ }^{2}$ Pei Cheng, ${ }^{1}$ Yang Pan, ${ }^{1}$ and Haiguang Wang ${ }^{1}$ \\ ${ }^{1}$ College of Plant Protection, China Agricultural University, Beijing 100193, China \\ ${ }^{2}$ College of Information and Electrical Engineering, China Agricultural University, Beijing 100083, China
}

Correspondence should be addressed to Haiguang Wang; wanghaiguang@cau.edu.cn

Received 15 April 2017; Accepted 27 June 2017; Published 6 August 2017

Academic Editor: Wee Chew

Copyright ( 2017 Yaqiong Zhao et al. This is an open access article distributed under the Creative Commons Attribution License, which permits unrestricted use, distribution, and reproduction in any medium, provided the original work is properly cited.

\begin{abstract}
Stripe rust caused by Puccinia striiformis f. sp. tritici (Pst) is a devastating wheat disease worldwide. Potential application of nearinfrared spectroscopy (NIRS) in detection of pathogen amounts in latently Pst-infected wheat leaves was investigated for disease prediction and control. A total of 300 near-infrared spectra were acquired from the Pst-infected leaf samples in an incubation period, and relative contents of Pst DNA in the samples were obtained using duplex TaqMan real-time PCR arrays. Determination models of the relative contents of Pst DNA in the samples were built using quantitative partial least squares (QPLS), support vector regression (SVR), and a method integrated with QPLS and SVR. The results showed that the $k$ QPLSSVR model built with a ratio of training set to testing set equal to $3: 1$ based on the original spectra, when the number of the randomly selected wavelength points was 700, the number of principal components was 8 , and the number of the built QPLS models was 5, was the best. The results indicated that quantitative detection of Pst DNA in leaves in the incubation period could be implemented using NIRS. A novel method for determination of latent infection levels of Pst and early detection of stripe rust was provided.
\end{abstract}

\section{Introduction}

Wheat stripe rust, caused by the biotrophic pathogen Puccinia striiformis f. sp. tritici (Pst), is an important wheat disease worldwide [1-5]. This disease can cause severe yield losses. Once an epidemic of this disease occurs, at least 10\%-30\% of wheat yield can be reduced [1]. In China, wheat stripe rust occurs in almost all wheat-growing regions, and it is the most devastating wheat disease $[1,4,5]$. This disease is always a great potential threat to the national wheat production in China. Since 1950, many severe epidemics of wheat stripe rust have occurred in China $[1,5]$, among which the four most destructive epidemics occurred in 1950, 1964, 1990, and 2002, resulting in yield losses of $6.0,3.2,1.8$, and 1.3 million tons, respectively $[4,6]$.

Generally, an infection process of wheat stripe rust in the field can be divided into four stages, that is, the contact period, the penetration period, the incubation period, and the diseased period [1]. Under favorable environmental conditions, Pst urediospores landing on the surface of wheat leaves germinate, produce germ tubes and appressoria, and then penetrate into wheat leaf tissues $[1,5]$. During the incubation period, a large quantity of Pst hyphae accumulate gradually in the infected leaf tissues $[1,5]$, but it is very difficult to observe disease symptoms on the surface of the infected wheat leaves with naked eyes, causing lots of difficulties for early monitoring and prediction of this disease. Once uredinia appear on the surface of wheat leaves, a large number of urediospores may be liberated after rupture of the uredinia and then be spread by air, which may result in secondary infections. So it is of great significance to conduct the early detection of Pst infections and the quantitative detection of pathogen amounts in the infected plants for early precise site-specific control of the disease, disease 
prediction, and control strategy making. In particular, it is very important to realize the early detection of pathogen amounts in the infected plants in the overwintering regions and the oversummering regions of Pst for the macro control of wheat stripe rust. Traditionally, the monitoring of wheat stripe rust is carried out via field investigation. This method is time-consuming and laborious. Moreover, using this conventional method, only the diseased wheat fields with disease symptoms can be surveyed, and the infected wheat leaves without symptom appearance in the incubation period cannot be identified accurately and rapidly. At present, some techniques and methods, including molecular biology techniques [7-10], hyperspectral remote sensing technology [11-14], thermal infrared imaging technology [15], and near-infrared spectroscopy (NIRS) [16, 17], have been applied to the monitoring and early detection of wheat stripe rust.

There are many reports on early qualitative detection of wheat stripe rust. The Pst infection of a wheat leaf in the incubation period of stripe rust can be detected accurately and qualitatively by detecting the presence of wheat stripe rust pathogen using molecular biology techniques such as polymerase chain reaction (PCR) assay and loop-mediated isothermal amplification (LAMP) assay [7-9]. Hyperspectral remote sensing technology has been used to identify healthy wheat plants and Pst-infected wheat plants without symptoms [18]. It was reported that Pst latent infections in wheat leaves could be detected using thermal infrared imaging technology [15] and NIRS technology [16, 17] before symptoms appear. A study on the detection of Pst latent infections in wheat leaves incubated in an artificial climate chamber using NIRS technology was conducted by Li et al. [17], and the results obtained by analyzing the spectral data demonstrated that the latently infected leaves could be distinguished from healthy wheat leaves as early as one day after artificial inoculation with the suspension of Pst urediospores. However, there are relatively few studies on the early quantitative detection of Pst in the incubation period of wheat stripe rust. The quantitative detection of Pst in the latently infected wheat leaves was implemented mainly relying on a realtime PCR method $[10,19]$.

The detection process using a molecular biological method is complex, and the method has high requirements on technologies and instruments $[20,21]$. With the development of science and technology, portable fluorescence quantitative PCR instruments have been applied gradually in recent years and the instruments for quantitative analysis in the field have been available [20]. However, it is still necessary for users to master the relevant molecular biology skills, and a large number of samples to be tested are required to be obtained. Only using molecular biology technology, it is very difficult to quickly get enough data in a large scale to provide information for disease forecasting and disease warning. The instruments used in hyperspectral remote sensing are very expensive. Thermal infrared imaging technology has high requirements on imaging resolution and temperature resolution of the used instrument. At the present time, detection methods of latent infection of pathogens based on molecular biology technology, hyperspectral remote sensing technology, and thermal infrared imaging technology are very difficult to be popularized in practice. Therefore, it is critical to explore a simple, convenient, accurate, and rapid method for early detection of wheat stripe rust.

As a kind of nondestructive analytical technology, NIRS can be used to carry out both qualitative analysis and quantitative analysis of samples [22, 23]. The analysis process of NIRS is simple and rapid, and it is a fast detection technology with low cost and is suitable for online analysis [22, 23]. It has been widely used in many fields such as agriculture, food industry, chemical industry, pharmaceutical industry, and petroleum industry [21-27]. NIRS has been applied to implement early qualitative identification of wheat stripe rust and wheat leaf rust (caused by Puccinia triticina) before disease symptoms appeared [16] and assess disease severity of wheat stripe rust [28]. It has also been used to qualitatively identify urediospores of Pst and P. triticina and quantitatively determine the content of each pathogen in a mixture of two kinds of pathogens including Pst and P. triticina [29]. Moreover, using NIRS, wheat leaves infected with Pst could be identified before symptoms appear [17]. However, to the best of our knowledge, there are no reports on quantitative detection of Pst in wheat leaves in the incubation period of wheat stripe rust using NIRS.

In this study, a method based on NIRS was explored for quantitative determination of the relative content of Pst DNA in wheat leaves in the incubation period. Based on the acquired near-infrared spectral data of the latently Pstinfected wheat leaf samples and the data on the relative content of Pst DNA in the corresponding samples obtained using the duplex TaqMan real-time PCR arrays, dynamic changes of the relative contents of Pst DNA in the latently Pstinfected wheat leaves during the incubation period were investigated and quantitative determination models were built to realize the quantitative and rapid detection of the pathogen amounts in the latently Pst-infected wheat leaves. The aim of this study was to provide a method for rapid, nondestructive, and quantitative determination of latent infection levels of Pst in wheat leaves and early detection of wheat stripe rust. Furthermore, support information can be provided for prediction and control of this disease. Some methodological references can also be provided for the early quantitative and nondestructive detection of other diseases.

\section{Materials and Methods}

2.1. Materials. CYR 33, a dominant physiological race of Pst in China, was used in this study. A highly susceptible wheat cultivar Mingxian 169 was used to multiply the pathogen in an artificial climate chamber in the Laboratory of Plant Disease Epidemiology, Department of Plant Pathology, China Agricultural University. Artificial inoculation by spraying urediospores of CYR 33 onto the surface of seedling leaves of Mingxian 169 was conducted to obtain the latently Pstinfected wheat leaves.

2.2. Multiplication of Wheat Stripe Rust Pathogen. Urediospores of the Pst physiological race CYR33 were multiplied using a similar method as described by Cheng et al. [30]. 


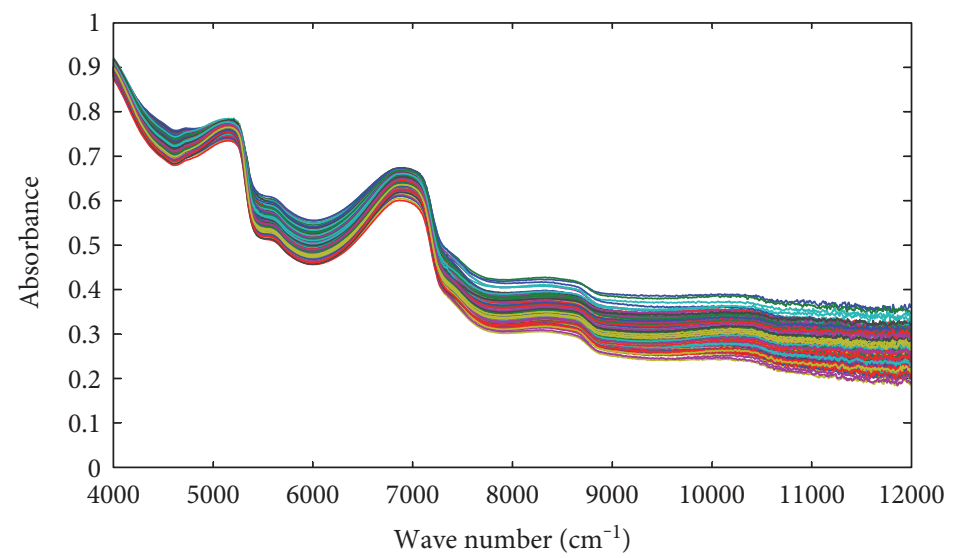

Figure 1: Near-infrared spectra of wheat leaves during the incubation period of wheat stripe rust.

After seeds of Mingxian 169 were soaked for $24 \mathrm{~h}$ in sterile water, the plump and well-germinated seeds were selected and sown in pots $(10 \mathrm{~cm}$ in diameter) with approximately 25 seeds per pot. Then the pots were incubated in an artificial climate chamber at $11-13^{\circ} \mathrm{C}$ with $60 \%-70 \%$ relative humidity (RH) and $14 \mathrm{~h}$ of light per day (10,000 lux) and were watered when necessary. When the first leaves of wheat seedlings fully expanded, urediospores of CYR 33 stored in a liquid nitrogen container were taken out, reactivated in warm water of 40$45^{\circ} \mathrm{C}$ for $5 \mathrm{~min}$, and then hydrated at $4^{\circ} \mathrm{C}$ for $12 \mathrm{~h}$. A spore suspension was made with $0.2 \%$ Tween 80 solution for artificial spray inoculation. Immediately after inoculation, each pot with the inoculated wheat seedlings was covered with a clear glass cylinder that was covered with two layers of sterile cotton gauze on the top, and all pots with the inoculated wheat seedlings were transferred into a moist chamber under dark conditions at $11-13^{\circ} \mathrm{C}$ for $24 \mathrm{~h}$. Subsequently, the inoculated wheat seedlings were placed into the artificial climate chamber under the conditions described above and incubated until a large number of urediospores were produced on the surface of the seedling leaves. The fresh urediospores were harvested for the following experiments or stored in the liquid nitrogen container for later use.

2.3. Collection of Latently Pst-Infected Wheat Leaves. Using the artificial spray inoculation method described above, healthy wheat seedlings with the fully expanded first leaves were inoculated with a $0.15 \mathrm{mg} / \mathrm{mL}$ spore suspension prepared with $3 \mathrm{mg}$ harvested fresh urediospores of CYR33 and $0.2 \%$ Tween 80 solution. A total of 50 pots of wheat seedlings were inoculated for further experiments. Sixty wheat leaves with uniform size and same growth vigor were collected every $24 \mathrm{~h}$ after inoculation until disease symptoms appeared and the incubation period of wheat stripe rust ended. The incubation period was defined as the number of days from inoculation to rupture of the first uredinium [31], and it was 10 days in this study. Two leaves were treated as a sample for acquisition of near-infrared spectral data, and 30 samples per day were collected. A total of 300 samples were collected in this study.
2.4. Acquisition of Near-Infrared Spectral Data. The nearinfrared spectra of the latently Pst-infected wheat leaf samples were acquired by using FT-NIR MPA spectrometer (Bruker, Germany). Wheat leaves of each sample were cut into small square fragments and then were placed into a sample cup (20 mm in diameter) for spectral measurement. Using integrating sphere diffuse reflectance method, the spectra in a range of $4000-12,000 \mathrm{~cm}^{-1}$ were measured with the spectral resolution of $8 \mathrm{~cm}^{-1}$ and the number of scan processes of 32. Each acquired spectrum contained 2100 wavelength points. After spectral acquisition, each sample was put into a $2 \mathrm{~mL}$ grinding tube. Each tube was numbered and immediately kept at $-80^{\circ} \mathrm{C}$ for subsequent DNA extraction. On each day during the incubation period, the nearinfrared spectra of 30 collected samples were acquired. In this study, a total of 300 spectra were obtained as shown in Figure 1.

2.5. DNA Extraction and Quantitative Measurements of DNA in Wheat Leaf Samples. Healthy wheat leaves, $3 \mathrm{mg}$ of urediospores of Pst, and the latently Pst-infected wheat leaves after acquisition of near-infrared spectral data were used as samples for DNA extraction. The extracted wheat DNA from the healthy leaves and the extracted Pst DNA from the Pst urediospores were used to generate standard curves. DNA was extracted using a modification of the procedure described by Justesen et al. [32]. Each sample in a $2 \mathrm{~mL}$ grinding tube was mixed with $0.4 \mathrm{~g}$ of quartz sands, one glass bead, and $600 \mu \mathrm{L}$ of $2 \%$ cetyl trimethyl ammonium bromide (CTAB) buffer. The CTAB buffer was prepared with $2 \mathrm{~g}$ of $\mathrm{CTAB}$ and $1 \mathrm{~g}$ of polyvinyl pyrrolidone by adding $10 \mu \mathrm{L}$ of $1 \mathrm{M}$ Tris- $\mathrm{HCl}(\mathrm{pH}=8.0), 2 \mu \mathrm{L}$ of $0.5 \mathrm{M}$ ethylene diamine tetraacetic acid $(\mathrm{pH}=8.0)$, and $28 \mathrm{~mL}$ of $5 \mathrm{M} \mathrm{NaCl}$ into a final volume of $100 \mathrm{~mL}$ adjusted with deionized water, then was sterilized under high temperature and high pressure, and finally was added with $100 \mu \mathrm{L} \beta$-mercapto ethanol after cooling. The tube was shaken in a FastPrep-24 instrument (MP Biomedicals, Santa Ana, CA, USA) at $6.0 \mathrm{~m} / \mathrm{s}$ for two periods of $40 \mathrm{~s}$, with $5 \mathrm{~min}$ cooling on ice between them. After incubation in water bath at $65^{\circ} \mathrm{C}$ for $1 \mathrm{~h}$ with gentle shakes every 
TAble 1: Duplex TaqMan real-time PCR primers and probes used in this study.

\begin{tabular}{lccc}
\hline Primer/probe & NCBI accession number & Sequence of primers and corresponding probes (5'-3') & Amplified fragment length (bp) \\
\hline TAG2315F & AF280605.1 & CAGAAAGCGAGTGGAAAGATGAAAG & 181 \\
TAG2473R & & GCAAGGAGGACAAAGATGAGGAA & \\
TAG-Pr1 & HEX-CAAGCATCAAAGGCAAGCAAGCAGTAGT-BHQ1 & 102 \\
Pst -F & GU382673.1 & AACCCTCTCATTAAATAATTTTG \\
$P s t$ - $\mathrm{C}$ & CCAACTTATAGAAAAGTGACTTA & \\
Pst P & FAM-ATTACAGCAGCACTCAACATCCATT-BHQ1 \\
\hline
\end{tabular}

$15 \mathrm{~min}$, the tube was added with $600 \mu \mathrm{L}$ of chloroform/ isoamyl alcohol $(v: v=24: 1)$ with mixing and then was centrifuged at the speed of $12,000 \mathrm{rpm}$ for $10 \mathrm{~min}$. The supernatant was transferred to a new clean $1.5 \mathrm{~mL}$ centrifuge tube, and 0.6 volumes of cold isopropanol at $-20^{\circ} \mathrm{C}$ was added. The mixture in the tube was shaken gently and then was incubated at $-20^{\circ} \mathrm{C}$ for $1 \mathrm{~h}$. The tube was centrifuged at the speed of $12,000 \mathrm{rpm}$ for $10 \mathrm{~min}$, and then the liquid supernatant was abandoned. The resultant was added with $500 \mu \mathrm{L}$ of $70 \%$ ethanol for rinse, shaken gently, and then centrifuged at $12000 \mathrm{rpm}$ for $10 \mathrm{~min}$. After discarding the supernatant and drying the resultant in the air, the DNA was dissolved in $50 \mu \mathrm{L}$ of sterile double distilled water and kept at $-20^{\circ} \mathrm{C}$ for later use.

Primers and probes used for the duplex TaqMan realtime PCR assays in this study were listed in Table 1. When the quantity of wheat DNA was determined using duplex TaqMan real-time PCR, TAG2315F and TAG2473R reported by Sandberg et al. [33] that were designed based on the DNA sequence of a prolamin gene of wheat were used as the primers, and the probe TAG-Pr1 was designed based on this DNA sequence. When the quantity of Pst DNA was determined using duplex TaqMan real-time PCR, the primers (Pst-F and Pst-R) and the probe Pst-P reported by Li et al. [34] that were designed based on the internal transcribed spacer (ITS) region sequence of Pst were used.

Each real-time PCR assay was performed in a volume of $20 \mu \mathrm{L}$ containing $3.20 \mu \mathrm{L}$ of $\mathrm{MgCl}_{2}(25 \mu \mathrm{M}), 2.00 \mu \mathrm{L}$ of dNTP $(2500 \mu \mathrm{M}), 2.00 \mu \mathrm{L}$ of Taq buffer (10x), $0.60 \mu \mathrm{L}$ of Taq (5 U/ $\mu \mathrm{L}), 0.30 \mu \mathrm{L}$ of each primer (Pst-F, Pst-R, TAG2315F, and TAG2473R, $10 \mu \mathrm{M}$ each), $0.25 \mu \mathrm{L}$ of each probe (Pst-P and TAG-Pr1, $10 \mu \mathrm{M}$ each), $2.00 \mu \mathrm{L}$ of template DNA, and double distilled water added to a final volume of $20 \mu \mathrm{L}$.

The real-time PCR amplification conditions were as follows: 1 cycle of initial denaturation at $95^{\circ} \mathrm{C}$ for $3 \mathrm{~min}$ and 40 cycles consisting of denaturation at $94^{\circ} \mathrm{C}$ for $20 \mathrm{~s}$, annealing at $56^{\circ} \mathrm{C}$ for $30 \mathrm{~s}$ and extension at $72^{\circ} \mathrm{C}$ for $30 \mathrm{~s}$ (fluorescence signal detection was conducted under this condition).

To generate standard curves, tenfold serial dilutions of Pst DNA $\left(10,1,10^{-1}, 10^{-2}, 10^{-3}\right.$, and $\left.10^{-4} \mathrm{ng} / \mu \mathrm{L}\right)$ and wheat DNA $\left(100,10,1,10^{-1}\right.$, and $\left.10^{-2} \mathrm{ng} / \mu \mathrm{L}\right)$ were made. The realtime PCR amplifications were conducted as described above, and the corresponding cycle threshold $(\mathrm{Ct})$ values were recorded. Then two standard curves to quantify Pst DNA and wheat DNA from the samples consisting of the latently Pst-infected wheat leaves after acquisition of near-infrared spectral data were generated.
Using the extracted DNA from the latently Pst-infected samples as template DNA, the real-time PCR amplifications were conducted as described above, and the corresponding Ct value of each sample was obtained. According to the standard curves, the contents of Pst DNA and wheat DNA were quantitatively determined. And the relative content of Pst DNA could be calculated using the following formula: $\mathrm{RCP}=\mathrm{CP} \times 100 \% /(\mathrm{CP}+\mathrm{CW})$, where $\mathrm{RCP}$ is the relative content of Pst DNA, CP is the content of Pst DNA (ng), and $\mathrm{CW}$ is the content of wheat DNA (ng).

2.6. Establishment of Determination Models for Quantification of the Relative Content of Pst DNA in Wheat Leaves in the Incubation Period. The obtained near-infrared spectra of the latently Pst-infected wheat leaf samples were preprocessed by using three methods including multiplication scatter correction (MSC), standard normalized variate (SNV), and vector normalization (VN). The 300 obtained spectra of the samples were divided into training set and testing set based on a ratio of the training set to the testing set equal to $3: 1$. By the combined use of quantitative partial least squares (QPLS) and support vector regression (SVR), the kQPLSSVR models to quantify the relative content of Pst DNA in wheat leaves in the incubation period were built based on the original near-infrared spectra and the spectral data obtained by using the three preprocessing methods.

As shown in Figure 2, to build a kQPLS-SVR model, firstly, $m$ features were randomly selected from the spectral features (spectral attributes) of 2100 wavelength points, the number of principal components was set as $n$, and a QPLS model was built. Using this method, $k$ QPLS models were built; secondly, a SVR model was built by using the predicted values of the $k$ QPLS models as variable. Thus, a $k$ QPLS-SVR model was obtained. The SVR model was built with radial basis function as the kernel function. Using the grid search algorithm, the penalty parameter $C$ and the kernel function parameter $g$ for the SVR model were optimized in a range of $2^{-8}-2^{8}$ with the searching step of 0.8 . As the minimum mean squared error of the training set was achieved at a point within the grid, the corresponding values of $C$ and $g$ were regarded as the optimal parameters for building the SVR model. In this study, the number of the randomly selected spectral features $(m)$ was set as 700 or 1400 , the number of principal components $(n)$ while building a QPLS model was set as 4,8 , or 12 , and the number of the built QPLS models (k) was set as 5, 10, or 15. All the calculations and modeling processes described above were implemented using the 


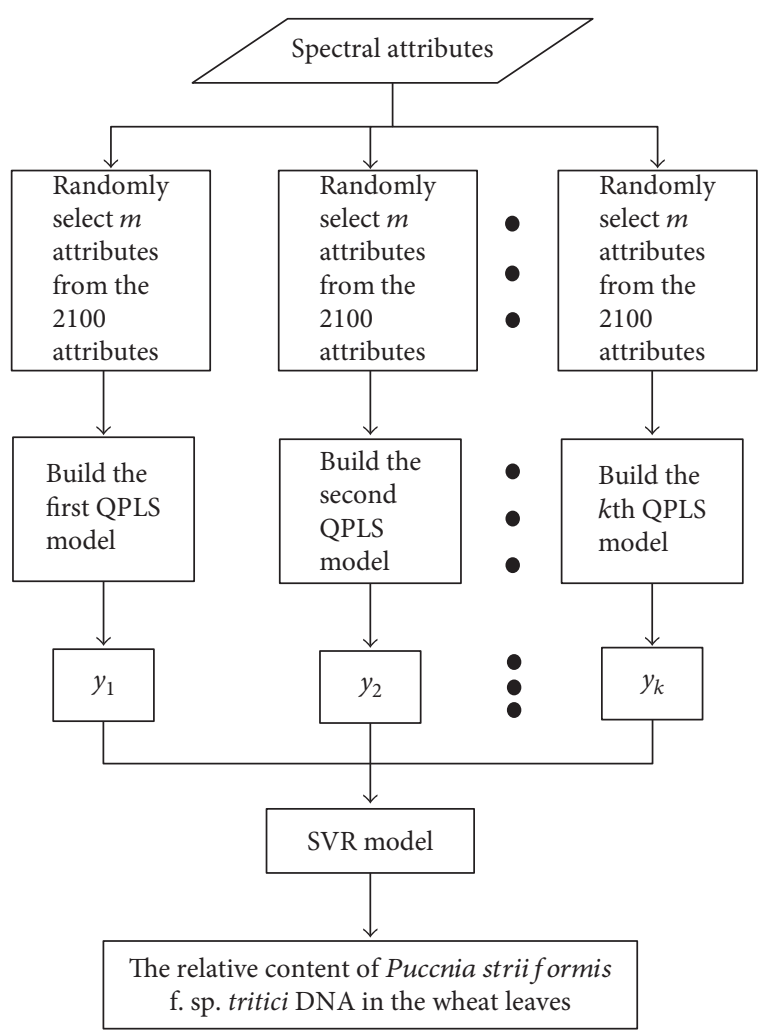

FIGURE 2: The algorithm flowchart for building the $k$ QPLS-SVR models to quantify the relative content of Pst DNA in wheat leaves in the incubation period.

software MATLAB 7.8.0 (R2009a) (MathWorks, Natick, MA, USA).

The coefficient of determination $\left(R^{2}\right)$, standard error of calibration (SEC), average absolute relative deviation (AARD) and relative prediction deviation (RPD) of the training set and $R^{2}$, standard error of prediction (SEP), AARD, and RPD of the testing set were used to evaluate the $k$ QPLS-SVR models built for quantification of the relative content of Pst DNA in wheat leaves in the incubation period. A value of $R^{2}$ more than 0.5 denotes that the corresponding model can be used for rough screening and actual application [35]. The closer the value of $R^{2}$ is to 1 , the higher the accuracy of the model is. The accuracy of a model is also related to the value of RPD, and a higher value of RPD denotes that the model has greater prediction ability [35]. The less value of SEC, SEP, or AARD denotes that higher accuracy can be obtained using the model and that the model has greater prediction ability. According to these evaluation indicators described above, the selection of the optimal $k$ QPLS-SVR model was conducted.

Determination models of the relative content of Pst DNA in wheat leaves in the incubation period were also built using individual methods including QPLS and SVR based on the same training set and testing set as used for building the optimal $k$ QPLS-SVR model. A comparison of the effects of the three models was conducted according to $R^{2}$, SEC, AARD, and RPD of the training set and $R^{2}$, SEP, AARD, and RPD of the testing set. The SVR model was built and optimized as described above. The number of principal components

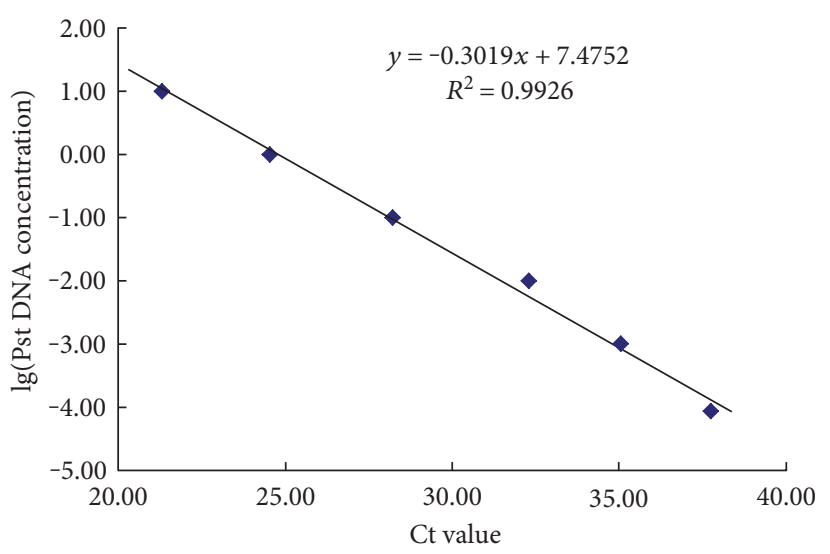

Figure 3: The standard curve of duplex TaqMan real-time PCR for quantification of Pst DNA and the corresponding linear regression equation.

used during building the QPLS model was determined by evaluating the prediction residual error sum of square (PRESS) [36]. Generally, when the minimum PRESS value is obtained, the value of the corresponding number of principal components may be the optimum, but it may lead to overfitting in this case. The value of PRESS can be calculated using the following formula [36]:

$$
\text { PRESS }=\sum_{i=1}^{n} \sum_{j=1}^{d}\left(y_{p, i j}-y_{i j}\right)^{2},
$$

where $n$ is the number of the samples in the training set, $d$ is the number of principal components used during modeling, $y_{p, i j}$ is the predicted value of the $i$ th sample of the training set, and $y_{i j}$ is the actual value of the $i$ th sample of the training set. In this study, the optimal number of principal components was determined using $F$ statistical method described as the following formula: $F(f)=\operatorname{PRESS}(f) / \operatorname{PRESS}\left(f^{*}\right)$, where $f^{*}$ is the number of principal components corresponding to the minimum PRESS value; the optimal number of principal components $(f)$ is less than $f^{*}$, and it should be as small as possible and satisfy the following condition: $F(f)<F_{\alpha, m, m}(\alpha=0.25, m$ is the number of degrees of freedom) [36].

\section{Results}

3.1. Quantitative Measurements of Pst DNA in Wheat Leaves in the Incubation Period Using Duplex TaqMan Real-Time PCR. After the real-time PCR amplifications were conducted with tenfold serial dilutions of Pst DNA, the standard curve for quantification of Pst DNA (Figure 3) was generated using common logarithmic value of the concentration of Pst DNA $(\lg ($ Pst DNA concentration $))$ as the ordinate and $\mathrm{Ct}$ value as the abscissa, and the corresponding linear regression equation was obtained. As shown in Figure 3, the equation of the standard curve for quantification of Pst DNA was as follows: $y=-0.3019 x+7.4752\left(R^{2}=0.9926\right)$, where $y$ is $\lg (P s t$ DNA concentration) and $x$ is the $\mathrm{Ct}$ value. After the real-time PCR amplifications were conducted with tenfold serial 


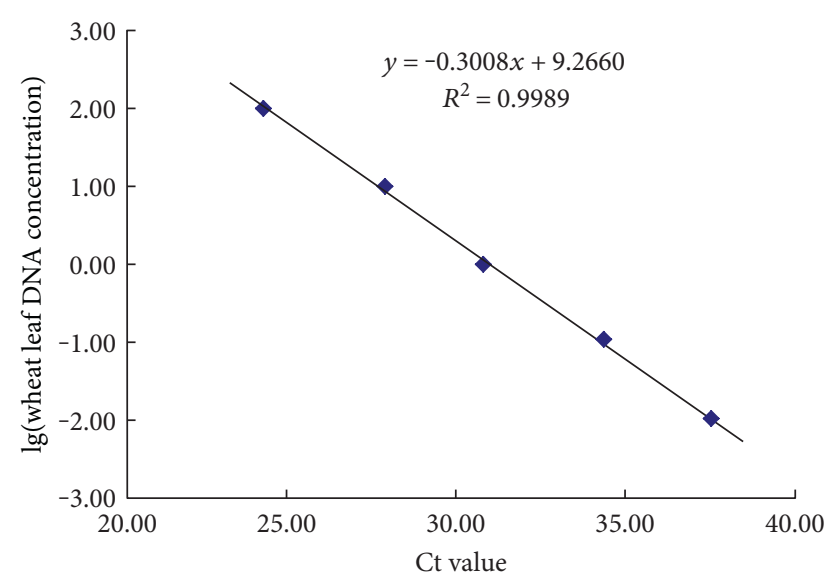

FIgURE 4: The standard curve of duplex TaqMan real-time PCR for quantification of wheat DNA and the corresponding linear regression equation.

dilutions of wheat DNA from healthy leaves, the standard curve for quantification of wheat DNA (Figure 4) was generated using common logarithmic value of the concentration of wheat DNA from leaves ( $\lg$ (wheat leaf DNA concentration)) as the ordinate and Ct value as the abscissa, and the corresponding linear regression equation was also obtained. As shown in Figure 4, the equation of the standard curve for quantification of wheat DNA was as follows: $y=-0.3008 x$ $+9.2660\left(R^{2}=0.9989\right)$, where $y$ is $\lg$ (wheat leaf DNA concentration) and $x$ is the Ct value. Moreover, satisfactory amplification efficiencies of the real-time PCR arrays were obtained for both Pst DNA and wheat DNA, and they were $99.7 \%$ and $100.1 \%$, respectively.

After the real-time PCR arrays were conducted with the extracted DNA from the latently Pst-infected wheat leaf samples, the contents of Pst DNA and wheat DNA in each sample were figured out based on the linear regression equations of the generated standard curves. The results showed that the relative content of Pst DNA increased exponentially with time (Figure 5). An equation to fit the daily changes of the relative contents of Pst DNA in wheat leaves after inoculation was built: $y=0.0096 e^{0.9287 x}\left(R^{2}=0.8784\right)$, where $y$ is the relative content of Pst DNA (\%) in a wheat leaf sample after inoculation and $x$ is the days post inoculation. In this study, attempts were made to build a linear regression model by using partial least squares method. The relative contents of Pst DNA in wheat leaves after inoculation changed in a range of $0.00385 \%-90.09 \%$. Especially, all relative contents of Pst DNA in the latently Pst-infected wheat leaves in the first six days were no more than $0.2 \%$. To make the data follow a normal distribution, a logarithmic transformation of the relative contents of Pst DNA in wheat leaves after inoculation was conducted in this study. Meanwhile, to ensure that each value after transformation was positive, each value of the relative contents of Pst DNA in wheat leaves after inoculation was multiplied by $10^{5}$ before the logarithmic transformation. The linear regression model $(y=0.4036 x+0.9861$, where $y$ is $\lg \left(\right.$ relative content Pst DNA $\left.\times 10^{5}\right)$ and $x$ is the days post inoculation) built based on the common logarithmic values

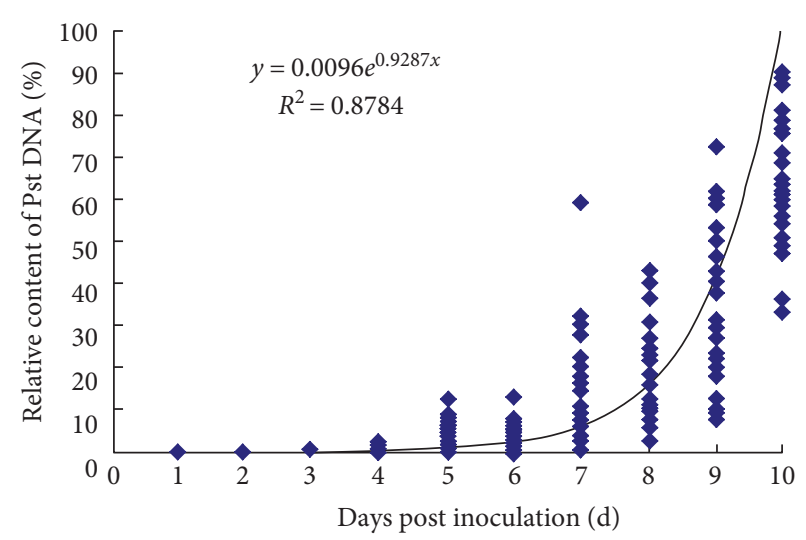

FIgURE 5: The daily changes of the relative contents of Pst DNA in wheat leaves after inoculation.

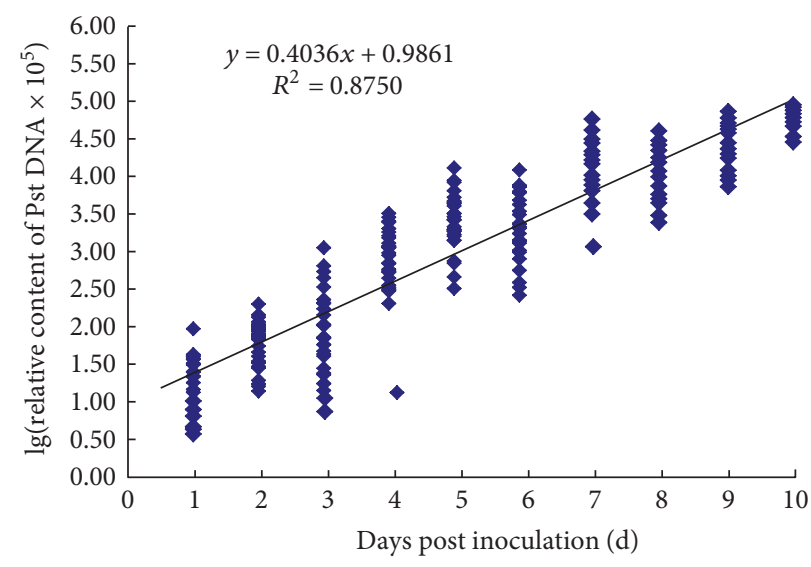

FIgURE 6: The daily changes of the common logarithmic values after transformation of the relative contents of Pst DNA in wheat leaves after inoculation.

was shown in Figure 6. As shown in Figures 5 and 6, the coefficients of determination $\left(R^{2}\right)$ of the equations built with the days post inoculation as independent variable before and after logarithmic transformation were 0.8784 and 0.8750 , respectively. It was demonstrated that there was no great difference between the values of $R^{2}$ of the two equations. Therefore, in combination with the near-infrared spectral data, $\lg \left(\mathrm{CP} \times 100 \% /(\mathrm{CP}+\mathrm{CW}) \times 10^{5}\right)$ in which $\mathrm{CP}$ was the content of $P s t$ DNA in ng and CW was the content of wheat DNA in ng, that is, $\lg \left(\right.$ relative content Pst DNA $\times 10^{5}$ ) was treated as chemical value for further modeling to develop a determination model for quantification of the relative content of Pst DNA in wheat leaves in the incubation period.

3.2. Results of the KQPLS-SVR Models for Quantification of the Relative Content of Pst DNA in Wheat Leaves in the Incubation Period. To quantitatively determine relative content of Pst DNA in wheat leaves in the incubation period using NIRS technology, the results of the $k$ QPLS-SVR models built with original near-infrared spectra were shown in Table 2 . The results demonstrated that the best effects were achieved for the kQPLS-SVR model built when the number 
TABLE 2: The results of the $k$ QPLS-SVR models for quantification of the relative content of Pst DNA in wheat leaves in the incubation period built with the original near-infrared spectra.

\begin{tabular}{|c|c|c|c|c|c|c|c|c|c|c|}
\hline \multirow{2}{*}{$\begin{array}{l}\text { The number } \\
\text { of attributes }\end{array}$} & \multirow{2}{*}{$\begin{array}{l}\text { The number of } \\
\text { principal components }\end{array}$} & \multirow{2}{*}{$\begin{array}{l}\text { The number of } \\
\text { QPLS models }\end{array}$} & \multicolumn{4}{|c|}{ Training set } & \multicolumn{4}{|c|}{ Testing set } \\
\hline & & & $R^{2}$ & SEC & AARD & RPD & $R^{2}$ & SEP & AARD & RPD \\
\hline 700 & 4 & 5 & 0.7179 & 0.6559 & 0.2283 & 1.8826 & 0.6773 & 0.7190 & 0.2624 & 1.7604 \\
\hline 700 & 4 & 10 & 0.7664 & 0.5968 & 0.1847 & 2.0692 & 0.7147 & 0.6760 & 0.2391 & 1.8723 \\
\hline 700 & 4 & 15 & 0.7729 & 0.5884 & 0.1836 & 2.0986 & 0.6732 & 0.7236 & 0.2645 & 1.7492 \\
\hline 700 & 8 & 5 & 0.9027 & 0.3852 & 0.1257 & 3.2057 & 0.8820 & 0.4349 & 0.1274 & 2.9107 \\
\hline 700 & 8 & 10 & 0.9029 & 0.3849 & 0.1282 & 3.2085 & 0.8788 & 0.4406 & 0.1400 & 2.8727 \\
\hline 700 & 8 & 15 & 0.9026 & 0.3854 & 0.1263 & 3.2042 & 0.8802 & 0.4382 & 0.1353 & 2.8888 \\
\hline 700 & 12 & 5 & 0.9564 & 0.2579 & 0.0872 & 4.7879 & 0.8514 & 0.4880 & 0.1458 & 2.5938 \\
\hline 700 & 12 & 10 & 0.9580 & 0.2531 & 0.0816 & 4.8797 & 0.8604 & 0.4729 & 0.1416 & 2.6767 \\
\hline 700 & 12 & 15 & 0.9590 & 0.2501 & 0.0808 & 4.9374 & 0.8535 & 0.4844 & 0.1414 & 2.6128 \\
\hline 1400 & 4 & 5 & 0.7193 & 0.6543 & 0.2158 & 1.8874 & 0.6309 & 0.7689 & 0.2636 & 1.6461 \\
\hline 1400 & 4 & 10 & 0.7047 & 0.6711 & 0.2173 & 1.8401 & 0.6422 & 0.7571 & 0.2598 & 1.6718 \\
\hline 1400 & 4 & 15 & 0.7729 & 0.5884 & 0.1836 & 2.0986 & 0.6732 & 0.7236 & 0.2645 & 1.7492 \\
\hline 1400 & 8 & 5 & 0.9072 & 0.3762 & 0.1241 & 3.2826 & 0.8806 & 0.4373 & 0.1342 & 2.8944 \\
\hline 1400 & 8 & 10 & 0.9039 & 0.3827 & 0.1273 & 3.2266 & 0.8771 & 0.4437 & 0.1385 & 2.8530 \\
\hline 1400 & 8 & 15 & 0.9036 & 0.3834 & 0.1284 & 3.2209 & 0.8785 & 0.4411 & 0.1356 & 2.8692 \\
\hline 1400 & 12 & 5 & 0.9668 & 0.2249 & 0.0739 & 5.4900 & 0.8180 & 0.5400 & 0.1634 & 2.3440 \\
\hline 1400 & 12 & 10 & 0.9666 & 0.2256 & 0.0751 & 5.4742 & 0.8215 & 0.5347 & 0.1572 & 2.3672 \\
\hline 1400 & 12 & 15 & 0.9659 & 0.2280 & 0.0739 & 5.4164 & 0.8223 & 0.5335 & 0.1555 & 2.3723 \\
\hline
\end{tabular}

TABLE 3: The results of the $k$ QPLS-SVR models for quantification of the relative content of Pst DNA in wheat leaves in the incubation period built when MSC was used as the preprocessing method of the original near-infrared spectra.

\begin{tabular}{lcccccccccc}
\hline $\begin{array}{l}\text { The number } \\
\text { of attributes }\end{array}$ & $\begin{array}{c}\text { The number of } \\
\text { principal components }\end{array}$ & $\begin{array}{c}\text { The number of } \\
\text { QPLS models }\end{array}$ & $R^{2}$ & SEC & AARD & RPD & \multicolumn{4}{c}{ Testing set } \\
\hline 700 & 4 & 5 & 0.8674 & 0.4497 & 0.1427 & 2.7460 & 0.8640 & 0.4668 & 0.1485 & 2.7117 \\
700 & 4 & 10 & 0.8792 & 0.4292 & 0.1325 & 2.8769 & 0.8689 & 0.4582 & 0.1464 & 2.7623 \\
700 & 4 & 15 & 0.9081 & 0.3744 & 0.1070 & 3.2980 & 0.8694 & 0.4574 & 0.1420 & 2.7674 \\
700 & 8 & 5 & 0.9215 & 0.3460 & 0.1148 & 3.5691 & 0.8712 & 0.4542 & 0.1367 & 2.7869 \\
700 & 8 & 10 & 0.9238 & 0.3408 & 0.1105 & 3.6234 & 0.8710 & 0.4547 & 0.1372 & 2.7838 \\
700 & 8 & 15 & 0.9227 & 0.3434 & 0.1127 & 3.5961 & 0.8730 & 0.4510 & 0.1361 & 2.8062 \\
700 & 12 & 5 & 0.9649 & 0.2312 & 0.0780 & 5.3402 & 0.8338 & 0.5160 & 0.1552 & 2.4530 \\
700 & 12 & 10 & 0.9645 & 0.2326 & 0.0737 & 5.3083 & 0.8534 & 0.4846 & 0.1444 & 2.6117 \\
700 & 12 & 15 & 0.9650 & 0.2311 & 0.0753 & 5.3430 & 0.8395 & 0.5071 & 0.1462 & 2.4962 \\
1400 & 4 & 5 & 0.8726 & 0.4408 & 0.1320 & 2.8013 & 0.8606 & 0.4725 & 0.1467 & 2.6786 \\
1400 & 4 & 10 & 0.8750 & 0.4367 & 0.1304 & 2.8280 & 0.8586 & 0.4760 & 0.1416 & 2.6590 \\
1400 & 4 & 15 & 0.9019 & 0.3869 & 0.1111 & 3.1922 & 0.8652 & 0.4648 & 0.1452 & 2.7232 \\
1400 & 8 & 5 & 0.9272 & 0.3332 & 0.1100 & 3.7056 & 0.8592 & 0.4750 & 0.1442 & 2.6648 \\
1400 & 8 & 10 & 0.9257 & 0.3366 & 0.1159 & 3.6688 & 0.8613 & 0.4713 & 0.1403 & 2.6855 \\
1400 & 8 & 15 & 0.9257 & 0.3366 & 0.1156 & 3.6683 & 0.8634 & 0.4677 & 0.1407 & 2.7060 \\
1400 & 12 & 5 & 0.9709 & 0.2108 & 0.0686 & 5.8573 & 0.7932 & 0.5756 & 0.1653 & 2.1990 \\
1400 & 12 & 10 & 0.9722 & 0.2058 & 0.0636 & 6.0017 & 0.7911 & 0.5785 & 0.1675 & 2.1881 \\
1400 & 12 & 15 & 0.9718 & 0.2073 & 0.0645 & 5.9559 & 0.7929 & 0.5760 & 0.1673 & 2.1973 \\
\hline & & & & & & & & &
\end{tabular}

of the spectral attributes of the randomly selected wavelength points was 700, the number of principal components was 8 , and the number of the built QPLS models was 5. For this model, the $R^{2}$ value, SEC, AARD, and RPD of the training set were 0.9027, 0.3852, 0.1257, and 3.2057, respectively, and the $R^{2}$ value, SEP, AARD, and RPD of the testing set were 0.8820, 0.4349, 0.1274, and 2.9107, respectively.

The results of the $k$ QPLS-SVR models built based on the data obtained after preprocessing of original near-infrared 
TABLE 4: The results of the kQPLS-SVR models for quantification of the relative content of Pst DNA in wheat leaves in the incubation period built when SNV was used as the preprocessing method of the original near-infrared spectra.

\begin{tabular}{lcccccccccc}
\hline $\begin{array}{l}\text { The number } \\
\text { of attributes }\end{array}$ & $\begin{array}{c}\text { The number of } \\
\text { principal components }\end{array}$ & $\begin{array}{c}\text { The number of } \\
\text { QPLS models }\end{array}$ & $R^{2}$ & Training set & \multicolumn{4}{c}{ Testing set } \\
\hline 700 & 4 & 5 & 0.8800 & 0.4278 & 0.1337 & 2.8863 & 0.8721 & 0.4527 & 0.1418 & 2.7959 \\
700 & 4 & 10 & 0.9009 & 0.3747 & 0.1069 & 3.2959 & 0.8643 & 0.4663 & 0.1501 & 2.7144 \\
700 & 4 & 15 & 0.9080 & 0.3747 & 0.1051 & 3.2961 & 0.8683 & 0.4593 & 0.1446 & 2.7560 \\
700 & 8 & 5 & 0.9255 & 0.3370 & 0.1096 & 3.6641 & 0.8725 & 0.4519 & 0.1320 & 2.8009 \\
700 & 8 & 10 & 0.9186 & 0.3522 & 0.1161 & 3.5058 & 0.8725 & 0.4520 & 0.1389 & 2.8005 \\
700 & 8 & 15 & 0.9225 & 0.3439 & 0.1151 & 3.5912 & 0.8712 & 0.4543 & 0.1379 & 2.7862 \\
700 & 12 & 5 & 0.9636 & 0.2356 & 0.0757 & 5.2422 & 0.8393 & 0.5074 & 0.1504 & 2.4947 \\
700 & 12 & 10 & 0.9660 & 0.2275 & 0.0723 & 5.4270 & 0.8456 & 0.4973 & 0.1486 & 2.5450 \\
700 & 12 & 15 & 0.9660 & 0.2276 & 0.0719 & 5.4257 & 0.8462 & 0.4963 & 0.1476 & 2.5503 \\
1400 & 4 & 5 & 0.8744 & 0.4376 & 0.1376 & 2.8217 & 0.8544 & 0.4831 & 0.1461 & 2.6203 \\
1400 & 4 & 10 & 0.8821 & 0.4241 & 0.1263 & 2.9120 & 0.8632 & 0.4681 & 0.1439 & 2.7039 \\
1400 & 4 & 15 & 0.8963 & 0.3976 & 0.1117 & 3.1057 & 0.8870 & 0.4255 & 0.1319 & 2.9748 \\
1400 & 8 & 5 & 0.9257 & 0.3367 & 0.1125 & 3.6676 & 0.8614 & 0.4712 & 0.1437 & 2.6861 \\
1400 & 8 & 10 & 0.9283 & 0.3307 & 0.1083 & 3.7346 & 0.8604 & 0.4729 & 0.1423 & 2.6763 \\
1400 & 8 & 15 & 0.9273 & 0.3330 & 0.1102 & 3.7087 & 0.8626 & 0.4693 & 0.1394 & 2.6973 \\
1400 & 12 & 5 & 0.9705 & 0.2122 & 0.0695 & 5.8193 & 0.7924 & 0.5768 & 0.1739 & 2.1945 \\
1400 & 12 & 10 & 0.9718 & 0.2074 & 0.0654 & 5.9552 & 0.7958 & 0.5720 & 0.1626 & 2.2128 \\
1400 & 12 & 15 & 0.9722 & 0.2060 & 0.0646 & 5.9937 & 0.7930 & 0.5759 & 0.1658 & 2.1978 \\
\hline & & & & & & & & & & \\
\hline
\end{tabular}

spectra by using MSC were shown in Table 3. The model built when the number of the spectral attributes randomly selected was 700, the number of principal components was 8 , and the number of the built QPLS models was 15 was better than others among the kQPLS-SVR models as shown in Table 3. For this model, the $R^{2}$ value, SEC, AARD, and RPD of the training set were $0.9227,0.3434,0.1127$, and 3.5961 , respectively, and the $R^{2}$ value, SEP, AARD, and RPD of the testing set were $0.8730,0.4510,0.1361$, and 2.8062 , respectively.

When the original near-infrared spectra were preprocessed by using the method SNV, the results of the built kQPLS-SVR models were shown in Table 4. Among the kQPLS-SVR models as shown in Table 4, the better effects were obtained for the model built when the number of the spectral attributes randomly selected was 1400 , the number of principal components was 4, and the number of the built QPLS models was 15. For this model, $R^{2}$, SEC, AARD, and RPD of the training set were $0.8963,0.3976,0.1117$, and 3.1057, respectively, and $R^{2}, \mathrm{SEP}, \mathrm{AARD}$, and RPD of the testing set were $0.8870,0.4255,0.1319$, and 2.9748, respectively.

The results of the built $k$ QPLS-SVR models based on the data obtained when $\mathrm{VN}$ was used as the preprocessing method were shown in Table 5. As demonstrated in Table 5, the effects of the model built when the number of the spectral attributes randomly selected was 700, the number of principal components was 8 , and the number of the built QPLS models was 15 was better than others. For this $k$ QPLS-SVR model, the value of $R^{2}$, SEC, AARD, and RPD of the training set were 0.8232, 0.5193, 0.1814, and 2.3782, respectively, and the value of $R^{2}$, SEP, AARD, and RPD of the testing set were $0.7964,0.5711,0.1833$, and 2.2164, respectively.

The results shown in Tables 2-5 indicated that satisfactory effects could be obtained using the kQPLS-SVR models built based on the original near-infrared spectra and the spectral data obtained by using MSC and SNV. In contrast, for the built $k$ QPLS-SVR models when the original nearinfrared spectra were preprocessed by using the method $\mathrm{VN}$, the values of both $R^{2}$ and RPD of the training set and the testing set were less. For the optimal $k$ QPLS-SVR model built based on the data obtained when SNV was used as the preprocessing method, the number of principal components was 4 . It was less than the number of principal components used for building the optimal $k$ QPLS-SVR model based on the original near-infrared spectra, the spectral data obtained by using MSC, or the spectral data obtained by using VN. In this case, there may be underfitting problem resulting in the reduction of the prediction ability of the optimal $k$ QPLS-SVR model built when SNV was used as the preprocessing method. For the optimal kQPLS-SVR model built based on the data obtained when MSC was used as the preprocessing method, the value of $R^{2}$ of the testing set was 0.8730 . And it was less than the value of $R^{2}$ of the testing set resulting from the optimal $k$ QPLS-SVR model built based on the original near-infrared spectra. Therefore, the optimal kQPLS-SVR model built based on the original near-infrared spectra (the number of the spectral attributes of the randomly selected wavelength points was 700 , the number of principal components was 8 , and the number of the built QPLS models was 5) was regarded as the optimal kQPLSSVR model to quantitatively determine the relative content of Pst DNA in wheat leaves in the incubation period. 
TABLE 5: The results of the $k$ QPLS-SVR models for quantification of the relative content of Pst DNA in wheat leaves in the incubation period built when $\mathrm{VN}$ was used as the preprocessing method of the original near-infrared spectra.

\begin{tabular}{|c|c|c|c|c|c|c|c|c|c|c|}
\hline \multirow{2}{*}{$\begin{array}{l}\text { The number of } \\
\text { attributes }\end{array}$} & \multirow{2}{*}{$\begin{array}{c}\text { The number of principal } \\
\text { components }\end{array}$} & \multirow{2}{*}{$\begin{array}{c}\text { The number of QPLS } \\
\text { models }\end{array}$} & \multicolumn{4}{|c|}{ Training set } & \multicolumn{4}{|c|}{ Testing set } \\
\hline & & & $R^{2}$ & SEC & AARD & RPD & $R^{2}$ & SEP & AARD & $\mathrm{RPD}$ \\
\hline 700 & 4 & 5 & 0.7040 & 0.6718 & 0.2424 & 1.8381 & 0.5965 & 0.8040 & 0.2667 & 1.5743 \\
\hline 700 & 4 & 10 & 0.6885 & 0.6893 & 0.2360 & 1.7916 & 0.6004 & 0.8001 & 0.2704 & 1.5820 \\
\hline 700 & 4 & 15 & 0.7313 & 0.6401 & 0.2186 & 1.9291 & 0.5987 & 0.8018 & 0.2584 & 1.5786 \\
\hline 700 & 8 & 5 & 0.8071 & 0.5424 & 0.1939 & 2.2766 & 0.7907 & 0.5790 & 0.1851 & 2.1860 \\
\hline 700 & 8 & 10 & 0.8247 & 0.5171 & 0.1826 & 2.3881 & 0.7784 & 0.5959 & 0.1967 & 2.1241 \\
\hline 700 & 8 & 15 & 0.8232 & 0.5193 & 0.1814 & 2.3782 & 0.7964 & 0.5711 & 0.1833 & 2.2164 \\
\hline 700 & 12 & 5 & 0.8522 & 0.4748 & 0.1718 & 2.6009 & 0.7556 & 0.6257 & 0.2033 & 2.0229 \\
\hline 700 & 12 & 10 & 0.8513 & 0.4762 & 0.1731 & 2.5934 & 0.7333 & 0.6540 & 0.2163 & 1.9353 \\
\hline 700 & 12 & 15 & 0.8697 & 0.4458 & 0.1531 & 2.7703 & 0.7475 & 0.6361 & 0.2063 & 1.9899 \\
\hline 1400 & 4 & 5 & 0.6789 & 0.6998 & 0.2459 & 1.7647 & 0.5489 & 0.8501 & 0.2706 & 1.4889 \\
\hline 1400 & 4 & 10 & 0.6974 & 0.6793 & 0.2350 & 1.8178 & 0.5382 & 0.8601 & 0.2803 & 1.4716 \\
\hline 1400 & 4 & 15 & 0.6804 & 0.6982 & 0.2488 & 1.7688 & 0.5324 & 0.8655 & 0.2836 & 1.4624 \\
\hline 1400 & 8 & 5 & 0.8220 & 0.5211 & 0.1827 & 2.3699 & 0.7578 & 0.6229 & 0.2001 & 2.0321 \\
\hline 1400 & 8 & 10 & 0.8136 & 0.5331 & 0.1902 & 2.3165 & 0.7668 & 0.6112 & 0.1987 & 2.0707 \\
\hline 1400 & 8 & 15 & 0.8095 & 0.5389 & 0.1949 & 2.2914 & 0.7625 & 0.6168 & 0.2050 & 2.0520 \\
\hline 1400 & 12 & 5 & 0.8565 & 0.4678 & 0.1629 & 2.6399 & 0.7078 & 0.6842 & 0.2295 & 1.8500 \\
\hline 1400 & 12 & 10 & 0.8695 & 0.4462 & 0.1526 & 2.7678 & 0.7479 & 0.6355 & 0.1947 & 1.9916 \\
\hline 1400 & 12 & 15 & 0.8717 & 0.4423 & 0.1498 & 2.7917 & 0.7234 & 0.6657 & 0.2118 & 1.9015 \\
\hline
\end{tabular}

TABLE 6: The results of the optimal models for quantification of the relative content of Pst DNA in wheat leaves in the incubation period built using individual methods including QPLS and SVR.

\begin{tabular}{|c|c|c|c|c|c|c|c|c|c|c|}
\hline \multirow{2}{*}{ Modeling methods } & \multicolumn{2}{|c|}{$\begin{array}{c}\text { Optimal } \\
\text { parameters }\end{array}$} & \multicolumn{4}{|c|}{ Training set } & \multicolumn{4}{|c|}{ Testing set } \\
\hline & $C$ & $g$ & $R^{2}$ & SEC & AARD & RPD & $R^{2}$ & SEP & AARD & RPD \\
\hline QPLS & - & - & 0.8534 & 0.4728 & 0.1689 & 2.6117 & 0.8684 & 0.4592 & 0.1467 & 2.7565 \\
\hline SVR & 256 & 0.1895 & 0.8887 & 0.4119 & 0.1277 & 2.9981 & 0.7971 & 0.5702 & 0.2110 & 2.2200 \\
\hline
\end{tabular}

3.3. Results of the QPLS Models and the SVR Model for Quantification of the Relative Content of Pst DNA in Wheat Leaves in the Incubation Period. Since the selected optimal $k$ QPLS-SVR model to quantitatively determine the relative content of Pst DNA in wheat leaves in the incubation period was built based on the original near-infrared spectra, the QPLS models and the SVR model were built with the original near-infrared spectra based on the same training set and testing set as used for building the optimal $k$ QPLS-SVR model. When the determination models of the relative content of Pst DNA in wheat leaves in the incubation period were built using QPLS, the number of principal components corresponding to the minimum PRESS value was calculated, and it was 8 . The value of $F_{\alpha, m, m}$ was calculated using the software IBM SPSS Statistics 19.0 (IBM Corporation, Somers, NY, USA), and it was 1.09. When the number of principal components was 6 , the value of $F(f)$ was 1.07. And the value of $F(f)$ was 1.02 when the number of principal components was 7. The two values of $F(f)$ were less than the value of $F_{\alpha, m, m}$. So the optimal number of principal components was set as 6 for building the QPLS model to quantitatively determine the relative content of Pst DNA in wheat leaves in the incubation period. As shown in Table 6, for the optimal QPLS model, the value of $R^{2}$, SEC, AARD, and RPD of the training set were $0.8534,0.4728,0.1689$, and 2.6117 , respectively, and the value of $R^{2}$, SEP, AARD, and RPD of the testing set were $0.8684,0.4592,0.1467$, and 2.7565 , respectively. When $C=256$ and $g=0.1895$, the optimal SVR model for quantification of the relative content of Pst DNA in wheat leaves in the incubation period was obtained. As shown in Table 6, for this SVR model, the value of $R^{2}$, SEC, AARD, and RPD of the training set were $0.8887,0.4119,0.1277$, and 2.9981, respectively, and the value of $R^{2}$, SEP, AARD, and RPD of the testing set were $0.7971,0.5702,0.2110$, and 2.2200 , respectively.

A comparison of the effects of the three models, including the optimal $k$ QPLS-SVR model, the optimal QPLS model, and the optimal SVR model, was conducted according to $R^{2}$, SEC, AARD, and RPD of the training set and $R^{2}$, SEP, $A A R D$, and RPD of the testing set. The results showed that the effects of the optimal $k$ QPLS-SVR model built based on the original near-infrared spectra (the number of the spectral attributes of the randomly selected wavelength points was 700 , the number of principal components was 8 , and the 
number of the built QPLS models was 5) were the best. Therefore, this optimal $k$ QPLS-SVR model was selected as the optimal model to quantitatively determine the relative content of Pst DNA in wheat leaves in the incubation period.

\section{Discussion}

For the $k$ QPLS-SVR models, the optimal QPLS model, and the optimal SVR model as shown in Tables $2-6$, all the values of $R^{2}$ of the training set and the testing set were more than 0.5 . The results indicated that the correlation between the features of near-infrared spectra (absorbances) and the relative content of Pst DNA in wheat leaves in the incubation period was relatively high and that the built models could be used for rough screening. It was indicated that quantitative determination of the relative content of Pst DNA in wheat leaves in the incubation period using NIRS technology is feasible. After a comprehensive comparison of the determination results of the models as shown in Tables 2-6, the $k$ QPLS-SVR model built with a ratio of the training set to the testing set equal to $3: 1$ based on the original nearinfrared spectra in a range of $4000-12,000 \mathrm{~cm}^{-1}$ when the number of the spectral attributes of the randomly selected wavelength points was 700 , the number of principal components was 8, and the number of the built QPLS models was 5 was regarded as the optimal model to quantitatively determine the relative content of Pst DNA in wheat leaves in the incubation period. The satisfactory effects were obtained using this optimal model. For this model, the value of $R^{2}$, SEC, AARD, and RPD of the training set were 0.9027, $0.3852,0.1257$, and 3.2057, respectively, and the value of $R^{2}$, SEP, AARD, and RPD of the testing set were 0.8820 , $0.4349,0.1274$, and 2.9107 , respectively. The results indicated that the proposed method based on NIRS could be used as a new method for rapid, nondestructive, and quantitative detection of Pst DNA content in wheat leaves. Meanwhile, a reference was provided for nondestructive determination of the DNA contents of other kinds of pathogens in plant hosts.

Changes of the near-infrared spectra of wheat leaves infected with Pst during the incubation period may be induced by many factors. The infection of $P s t$ and subsequent expansion in plant hosts are dynamic change processes $[1,5]$. After germination of a Pst urediospore, a growing germ tube emerges. Then an appressorium is formed at the tip of the germ tube. An infection peg grows from the appressorium and then enters the wheat leaf. During the incubation period, with the continuous spread of hyphae in the infected leaf tissues, changes of the photosynthesis, respiration, and transpiration of wheat leaves occur, affecting the synthesis and decomposition of organic substances [1,37-39]. Thus, the near-infrared spectra of the infected wheat leaf are influenced. Especially in the middle and later stage of the incubation period, a large quantity of Pst hyphae accumulate in the infected leaf tissues, the latent lesions on the infected leaves become more and more obvious, and the effects on various physiological indexes of wheat are increasing [1], more directly affecting the near-infrared spectra of the infected wheat leaves. In this study, according to the changes of the near-infrared spectra of the infected wheat leaves during the incubation period, the detection of the relative content of Pst DNA in the latently Pst-infected wheat leaves was performed using NIRS technology in combination with the duplex TaqMan real-time PCR arrays, providing a basis for modeling the quantitative relationship between the relative content of Pst DNA in the latently Pst-infected wheat leaves and the corresponding near-infrared spectral data.

The quantitative detection results obtained by using the duplex TaqMan real-time PCR arrays in this study showed that the quantity of Pst DNA exponentially increased as Pst extended in wheat leaf during the incubation period of wheat stripe rust, and it was consistent with the growth trend of Pst DNA reported by Pan et al. [10]. The results in this study showed that the relative contents of Pst DNA in wheat leaves after inoculation continuously increased. The relative contents of Pst DNA in the latently Pst-infected wheat leaves in the incubation period were more than $20 \%$ on 7 days post inoculation (dpi) and reached to approximately $90 \%$ on 10 dpi. It was demonstrated that there was a particularly obvious increase of the relative contents of Pst DNA in the latently Pst-infected wheat leaves from the seventh day to the tenth day during the incubation period. This indicated that it would have great significance to perform early detection and control of wheat stripe rust.

Wheat stripe rust is a kind of air-borne disease. As disease symptoms appear on wheat leaves, a large number of urediospores will be produced and serve as inocula for the further spread and epidemic of the disease [1,3-5]. Accurate quantitative determination of the latent infection levels of wheat leaves by Pst can provide important information for estimating potential inoculum level. Early detection of Pst infection, early disease warning, and early disease control measuremaking can effectively reduce the amount of inoculum sources and the dosage of pesticides. The results of this study demonstrated that latent infection of wheat seedlings caused by Pst could be detected as early as $24 \mathrm{~h}$ after inoculation based on NIRS. For the first time, dynamic changes of the amounts of wheat stripe rust pathogen in the infected leaves during the incubation period were investigated by using NIRS technology in combination with a real-time PCR method. A modeling method integrated with QPLS and SVR was used to establish the dynamic quantitative detection model of wheat stripe rust pathogen during the incubation period. A reference was provided for prediction of disease epidemic trend and early control of wheat stripe rust in this study.

\section{Conclusions}

It is critical to implement early quantitative detection of Pst and disease prediction accurately for the prevention and control of wheat stripe rust. In this study, a method based on NIRS to implement the quantitative determination of the relative content of Pst DNA in wheat leaves in the incubation period was investigated. Based on the data on the relative content of Pst DNA in wheat leaves in the incubation period obtained using the duplex TaqMan real-time PCR arrays and the corresponding near-infrared spectral data, the optimal model integrated with QPLS and SVR was obtained to 
quantify the relative content of Pst DNA in wheat leaves in the incubation period. Using this model, satisfactory results were achieved. The results indicated that rapid, nondestructive, and quantitative detection of the amount of Pst in the infected leaves during the incubation period could be realized using NIRS technology. A novel method for quantitatively estimating the latent infection levels of Pst in wheat leaves and early detection of wheat stripe rust was provided in this study. Furthermore, this proposed method can be used as a reference to establish models for determining the latent amounts of other kinds of pathogens in plant hosts and implement early nondestructive detection of the diseases caused by these agents. It is very helpful in getting the information about disease prevalence and inoculum amount in the field as early as possible, inhibiting pathogen accumulation, reducing the quantity of propagules, performing early precise site-specific control, disease forecasting, and making macro strategy for disease management. And it is also conducive to reducing the dosage of pesticides and increasing efficiency of control measures in plant disease management. Moreover, in this study, some basis was provided for the development of portable near-infrared spectrometers and sensors for quantitative detection of plant diseases.

\section{Conflicts of Interest}

The authors declare that there is no conflict of interests regarding the publication of this paper.

\section{Authors' Contributions}

Yaqiong Zhao, Yilin Gu, and Feng Qin contributed equally to this paper.

\section{Acknowledgments}

This study was supported by the National Key Basic Research Program of China (2013CB127700), National Natural Science Foundation of China (31471726), and National Key Technologies Research and Development Program of China (2012BAD19BA04).

\section{References}

[1] Z. Q. Li and S. M. Zeng, Wheat Rust in China, China Agriculture Press, Beijing, China, 2002.

[2] R. F. Line, "Stripe rust of wheat and barley in North America: a retrospective historical review," Annual Review of Phytopathology, vol. 40, pp. 75-118, 2002.

[3] X. M. Chen, "Epidemiology and control of stripe rust [Puccinia striiformis f. sp. tritici] on wheat," Canadian Journal of Plant Pathology, vol. 27, no. 3, pp. 314-337, 2005.

[4] A. M. Wan, X. M. Chen, and Z. H. He, "Wheat stripe rust in China," Australian Journal of Agricultural Research, vol. 58, no. 6, pp. 605-619, 2007.

[5] W. Chen, C. Wellings, X. Chen, Z. Kang, and T. Liu, "Wheat stripe (yellow) rust caused by Puccinia striiformis f. sp. tritici," Molecular Plant Pathology, vol. 15, no. 5, pp. 433-446, 2014.

[6] A. M. Wan, Z. H. Zhao, X. M. Chen et al., "Wheat stripe rust epidemic and virulence of Puccinia striiformis f. sp. tritici in
China in 2002," Plant Disease, vol. 88, no. 8, pp. 896-904, 2004.

[7] J. Zhao, X. J. Wang, C. Q. Chen, L. L. Huang, and Z. S. Kang, "A PCR-based assay for detection of Puccinia striiformis $\mathrm{f}$. sp. tritici in wheat," Plant Disease, vol. 91, no. 12, pp. 16691674, 2007.

[8] X. J. Wang, C. L. Tang, J. L. Chen et al., "Detection of Puccinia striiformis in latently infected wheat leaves by nested polymerase chain reaction," Journal of Phytopathology, vol. 157, no. 78, pp. 490-493, 2009.

[9] C. Huang, Z. Y. Sun, J. H. Yan, Y. Luo, H. G. Wang, and Z. H. $\mathrm{Ma}$, "Rapid and precise detection of latent infections of wheat stripe rust in wheat leaves using loop-mediated isothermal amplification," Journal of Phytopathology, vol. 159, no. 7-8, pp. 582-584, 2011.

[10] J. J. Pan, Y. Luo, C. Huang et al., "Quantification of latent infections of wheat stripe rust by using real-time PCR," Acta Phytopathologica Sinica, vol. 40, no. 4, pp. 504-510, 2010.

[11] D. Moshou, C. Bravo, J. West, S. Wahlen, A. McCartney, and H. Ramon, "Automatic detection of 'yellow rust' in wheat using reflectance measurements and neural networks," Computers and Electronics in Agriculture, vol. 44, no. 3, pp. 173188, 2004.

[12] R. Devadas, D. W. Lamb, S. Simpfendorfer, and D. Backhouse, "Evaluating ten spectral vegetation indices for identifying rust infection in individual wheat leaves," Precision Agriculture, vol. 10, no. 6, pp. 459-470, 2009.

[13] L. Yuan, Y. B. Huang, R. W. Loraamm, C. W. Nie, J. H. Wang, and J. C. Zhang, "Spectral analysis of winter wheat leaves for detection and differentiation of diseases and insects," Field Crops Research, vol. 156, pp. 199-207, 2014.

[14] J. L. Zhao, L. S. Huang, W. J. Huang et al., "Hyperspectral measurements of severity of stripe rust on individual wheat leaves," European Journal of Plant Pathology, vol. 139, no. 2, pp. 407417,2014

[15] X. L. Li, K. Wang, Z. H. Ma, and H. G. Wang, "Early detection of wheat disease based on thermal infrared imaging," Transactions of the Chinese Society of Agricultural Engineering, vol. 30, no. 18, pp. 183-189, 2014.

[16] X. L. Li, Z. H. Ma, L. L. Zhao, J. H. Li, and H. G. Wang, "Early diagnosis of wheat stripe rust and wheat leaf rust using near infrared spectroscopy," Spectroscopy and Spectral Analysis, vol. 33, no. 10, pp. 2661-2665, 2013.

[17] X. L. Li, F. Qin, L. L. Zhao, J. H. Li, Z. H. Ma, and H. G. Wang, "Detection of Puccinia striiformis f. sp. tritici latent infections in wheat leaves using near infrared spectroscopy technology," Spectroscopy and Spectral Analysis, vol. 34, no. 7, pp. 1853$1858,2014$.

[18] J. Li, Y. H. Chen, J. B. Jiang, and H. C. Cai, "Using hyperspectral derivative index to identify winter wheat stripe rust disease," Science \& Technology Review, vol. 25, no. 6, pp. 23-26, 2007.

[19] Y. Pan, Y. L. Gu, Y. Luo et al., "Study on relationship between the quantity of Puccinia striiformis f. sp. tritici in latent during overwinter and the disease index in Xiangyang area," Acta Phytopathologica Sinica, vol. 46, no. 5, pp. 679685, 2016.

[20] N. W. Schaad and R. D. Frederick, "Real-time PCR and its application for rapid plant disease diagnostics," Canadian Journal of Plant Pathology, vol. 24, no. 3, pp. 250-258, 2002. 
[21] S. Sankaran, A. Mishra, R. Ehsani, and C. Davis, "A review of advanced techniques for detecting plant diseases," Computers and Electronics in Agriculture, vol. 72, no. 1, pp. 1-13, 2010.

[22] G. T. Xu, H. F. Yuan, and W. Z. Lu, "Development of modern near infrared spectroscopic techniques and its applications," Spectroscopy and Spectral Analysis, vol. 20, no. 2, pp. 134$142,2000$.

[23] X. L. Chu and W. Z. Lu, "Research and application progress of near infrared spectroscopy analytical technology in China in the past five years," Spectroscopy and Spectral Analysis, vol. 34, no. 10, pp. 2595-2605, 2014.

[24] Q. S. Chen, J. W. Zhao, M. H. Liu, J. R. Cai, and J. H. Liu, "Determination of total polyphenols content in green tea using FT-NIR spectroscopy and different PLS algorithms," Journal of Pharmaceutical and Biomedical Analysis, vol. 46, no. 3, pp. 568-573, 2008.

[25] M. K. Ahmed and J. Levenson, "Application of near-infrared spectroscopy to the quality assurance of ethanol and butanol bended gasoline," Petroleum Science and Technology, vol. 30, no. 2, pp. 115-121, 2012.

[26] E. Tamburini, G. Ferrari, M. G. Marchetti, P. Pedrini, and S. Ferro, "Development of FT-NIR models for the simultaneous estimation of chlorophyll and nitrogen content in fresh apple (Malus domestica) leaves," Sensors, vol. 15, no. 2, pp. 26622679, 2015.

[27] H. Chen, Z. Lin, H. G. Wu, L. Wang, T. Wu, and C. Tan, "Diagnosis of colorectal cancer by near-infrared optical fiber spectroscopy and random forest," Spectrochimica Acta Part A: Molecular and Biomolecular Spectroscopy, vol. 135, pp. 185-191, 2015.

[28] X. L. Li, F. Qin, L. L. Zhao, J. H. Li, Z. H. Ma, and H. G. Wang, "Identification and classification of disease severity of wheat stripe rust using near infrared spectroscopy technology," Spectroscopy and Spectral Analysis, vol. 35, no. 2, pp. 367-371, 2015.

[29] X. L. Li, Z. H. Ma, L. L. Zhao, J. H. Li, and H. G. Wang, "Application of near infrared spectroscopy to qualitative identification and quantitative determination of Puccinia striiformis $\mathrm{f}$. sp. tritici and P. recondita f. sp. tritici," Spectroscopy and Spectral Analysis, vol. 34, no. 3, pp. 643-647, 2014.

[30] P. Cheng, Z. H. Ma, X. J. Wang et al., "Impact of UV-B radiation on aspects of germination and epidemiological components of three major physiological races of Puccinia striiformis f. sp. tritici," Crop Protection, vol. 65, pp. 6-14, 2014.

[31] Y. Y. Xiao and S. M. Zeng, "Comparisons among three equations to predict apparent infection rates of wheat stripe rust," Scientia Sinica (Series B), vol. 2, pp. 151-157, 1985.

[32] A. F. Justesen, C. J. Ridout, and M. S. Hovmoller, “The recent history of Puccinia striiformis f. sp. tritici in Denmark as revealed by disease incidence and AFLP markers," Plant Pathology, vol. 51, no. 1, pp. 13-23, 2002.

[33] M. Sandberg, L. Lundberg, M. Ferm, and I. M. Yman, "Real time PCR for the detection and discrimination of cereal contamination in gluten free foods," European Food Research and Technology, vol. 217, no. 4, pp. 344-349, 2003.

[34] Y. Li, Y. L. Gu, B. M. Wu et al., "Establishment of a duplex TaqMan real-time PCR method for quantifying Puccinia striiformis f. sp. tritici and Blumeria graminis f. sp. tritici," Acta Phytopathologica Sinica, vol. 45, no. 2, pp. 205-210, 2015.
[35] A. De Girolamo, S. Cervellieri, A. Visconti, and M. Pascale, "Rapid analysis of deoxynivalenol in durum wheat by FTNIR spectroscopy," Toxins, vol. 6, no. 11, pp. 3129-3143, 2014.

[36] W. Z. Lu, H. F. Yuan, G. T. Xu, and D. M. Qiang, Modern Near Infrared Spectroscopy Analytical Technology, China Petrochemical Press, Beijing, China, 2000.

[37] R. C. G. Smith, A. D. Heritage, M. Stapper, and H. D. Barrs, "Effect of stripe rust (Puccinia striiformis West.) and irrigation on the yield and foliage temperature of wheat," Field Crops Research, vol. 14, no. 1, pp. 39-51, 1986.

[38] Y. R. Li and H. S. Shang, "Effect of stripe rust infection on photosynthesis and transpiration of wheat," Journal of Triticeae Crops, vol. 21, no. 2, pp. 51-56, 2001.

[39] H. Zhao, R. Y. Wang, P. L. Ma, and J. L. Zhu, "Effect of stripe rust infection on photosynthesis and transpiration of wheat in the semi-arid region," Arid Meteorology, vol. 22, no. 4, pp. 5659, 2004. 

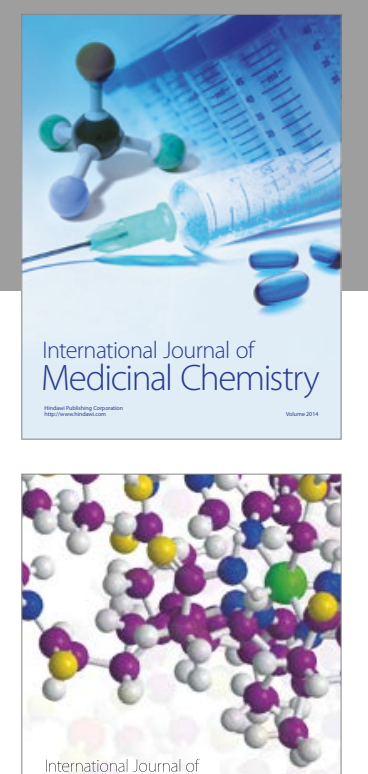

Carbohydrate Chemistry

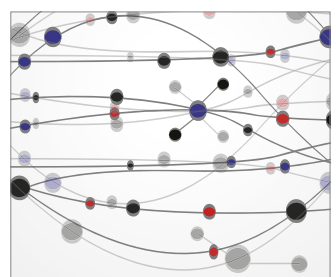

The Scientific World Journal
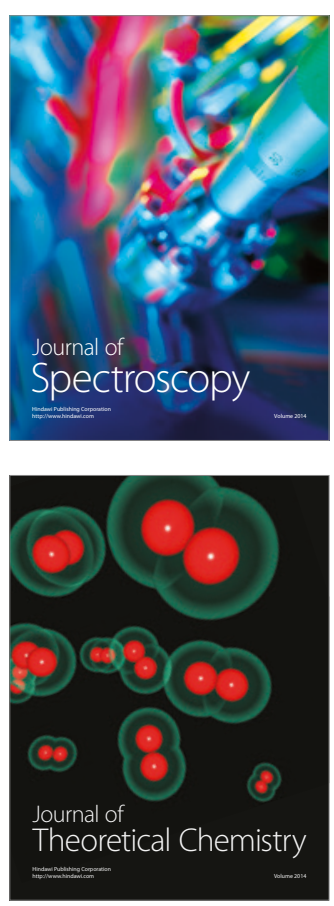
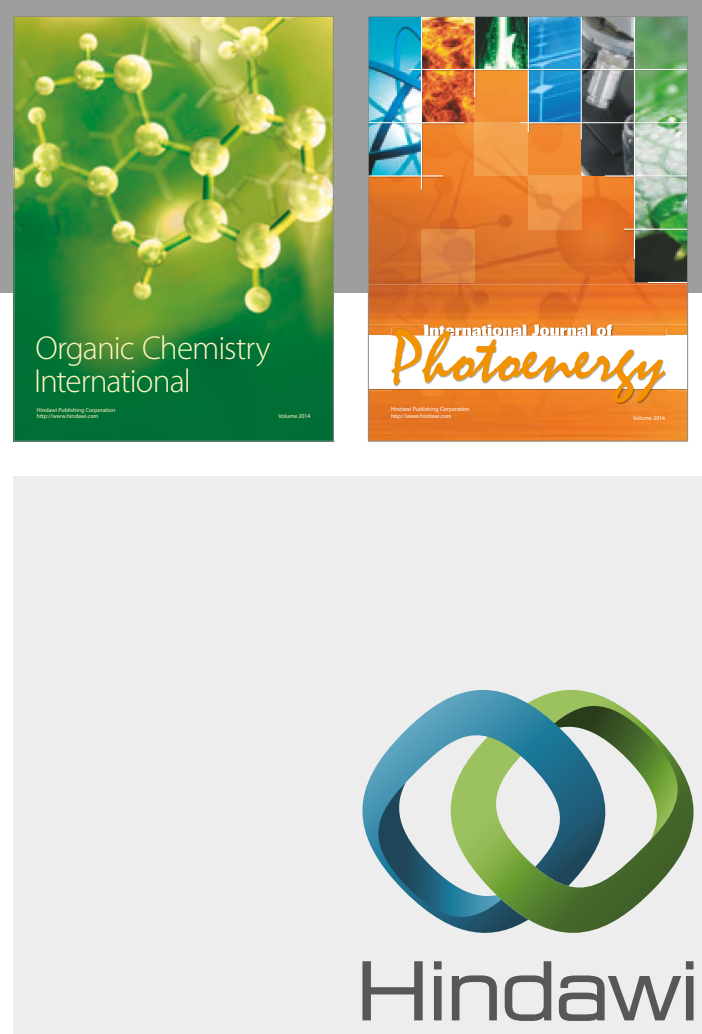

Submit your manuscripts at

https://www.hindawi.com

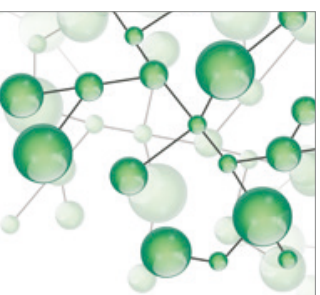

International Journal of

Inorganic Chemistry

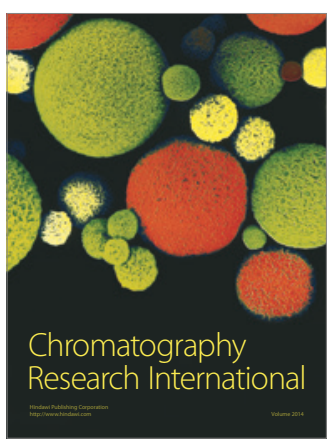

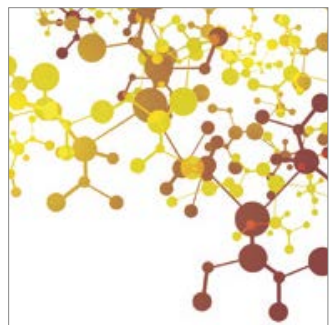

Applied Chemistry
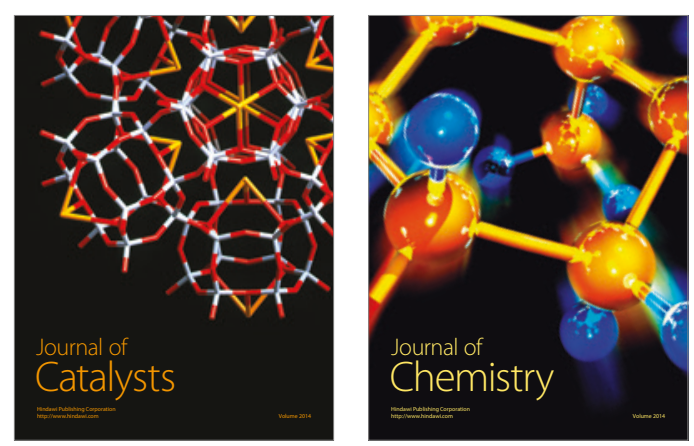
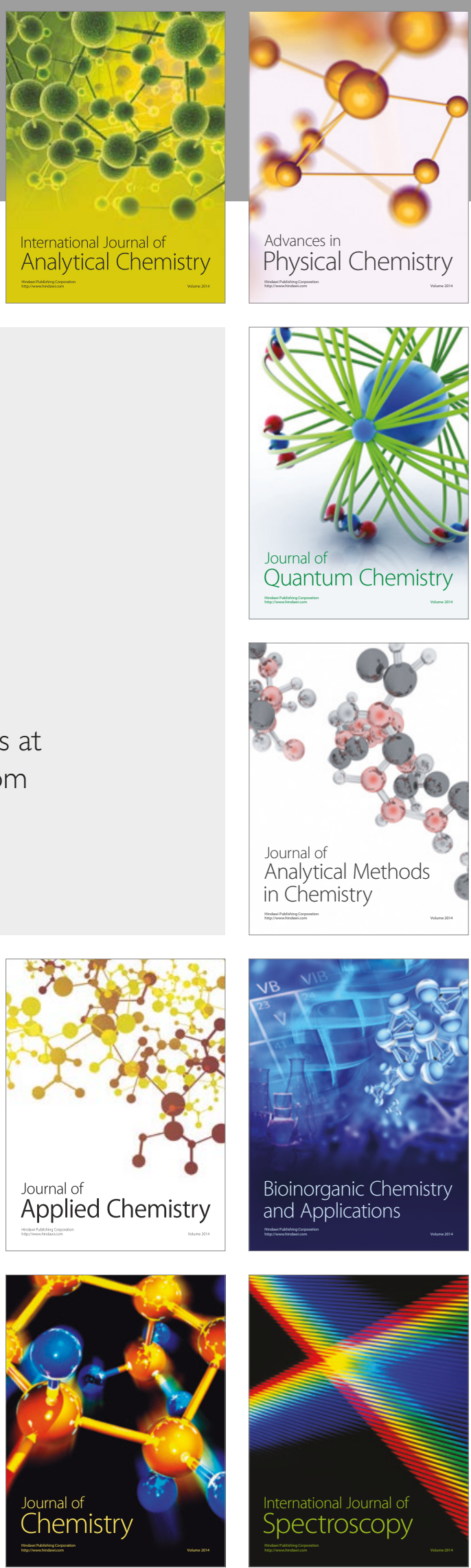